\title{
Transient eastward-propagating long-period waves observed over the South Pole
}

\author{
S. E. Palo ${ }^{1}$, Y. I. Portnyagin ${ }^{2}$, J. M. Forbes ${ }^{1}$, N. A. Makarov $^{2}$, and E. G. Merzlyakov ${ }^{2}$ \\ ${ }^{1}$ University of Colorado, Boulder, CO, USA \\ ${ }^{2}$ Institute for Experimental Meteorology, Obninsk, Russia
}

Received: 27 October 1997 / Revised: 9 March 1998 / Accepted 16 March 1998

\begin{abstract}
Observations of the horizontal wind field over the South Pole were made during 1995 using a meteor radar. These data have revealed the presence of a rich spectrum of waves over the South Pole with a distinct annual occurrence. Included in this spectrum are longperiod waves, whose periods are greater than one solar day, which are propagating eastward. These waves exhibit a distinct seasonal occurrence where the envelope of wave periods decreases from a period of 10 days near the fall equinox to a minimum of 2 days near the winter solstice and then progresses towards a period near 10 days at the spring equinox. Computation of the meridional gradient of quasi-geostrophic potential vorticity has revealed a region in the high-latitude upper mesosphere which could support an instability and serve as a source for these waves. Estimation of the wave periods which would be generated from an instability in this region closely resembles the observed seasonal variation in wave periods over the South Pole. These results are consistent with the hypothesis that the observed eastward propagating long-period waves over the South Pole are generated by an instability in the polar upper mesosphere. However, given our limited data set we cannot rule out a stratospheric source. Embedded in this spectrum of eastward propagating waves during the austral winter are a number of distinct wave events. Eight such wave events have been identified and localized using a constant- $Q$ filter bank. The periods of these wave events ranges from 1.7 to 9.8 days and all exist for at least 3 wave periods. Least squares analysis has revealed that a number of these events are inconsistent with a wave propagating zonally around the geographic pole and could be related to waves propagating around a dynamical pole which is offset from the geographic pole. Additionally, one event which was observed appears to be a standing oscillation.
\end{abstract}

Key words: Meteorology and atmospheric dynamics (Middle atmospheric dynamics; waves and tides).

\section{Introduction}

In November 1994 initial work began to install a meteor radar at Amundsen-Scott station, South Pole. The first measurements from this system began January 19, 1995 and continued through January 26, 1996 without interruption. A second continuous period of observations occurred from November 211996 to January 27, 1997. These meteor radar measurements are the first continuous measurements of the mesosphere and lowerthermospheric horizontal wind field over the South Pole and as a result have provided a picture of the dynamical structure in this region which was previously unknown. The pioneering work of Hernandez et al. (1992, 1993, 1995) at the South Pole provided an indication of the previously unknown dynamical structures which exist in this region. However, without the ability to make measurements during daytime it was not possible to infer the great dynamical variability which occurs over the South Pole on an annual basis.

Early results from our observations over the South Pole have confirmed the existence of a $12 \mathrm{~h}$ oscillation (Forbes et al., 1995; Portnyagin et al., 1997b) which was first observed by Collins et al. (1992) in sodium density fluctuations and then by Hernandez et al. (1993) in the meridional wind field. Additionally, a strong divergence event in April 1996 was observed and presented in Portnyagin et al. (1997a).

With uninterrupted measurements for one complete year in four different directions we have been able to characterize the annual occurrence of both eastward and westward propagating waves over the South Pole. There is a clear seasonal dependence with eastward propagating waves being observed primarily during winter and

Correspondence to: S. E. Palo 
strong 12 and $24 \mathrm{~h}$ westward propagating oscillations observed during the summer (Hernandez et al., 1993; Forbes et al., 1995; Portnyagin et al., 1997b). In this study we will focus on the eastward propagating longperiod waves which have been observed using the meteor radar over the South Pole. These waves have a period greater than one solar day. In the following sections we will discuss the meteor system and its limitations, then describe the seasonal occurrence of waves over the South Pole and finally conclude with a discussion of the observed transient long-period wave events over the South Pole.

\section{The meteor radar system}

The meteor radar which was used to make the neutral wind measurements presented here was described by Portnyagin et al. (1997a,b). To summarize, this system operates at a frequecy of $33.57 \mathrm{MHz}$, with a peak power of $7 \mathrm{~kW}$, a pulse width of $120 \mu$ s and a pulse repetition frequency of either 100 or $300 \mathrm{~Hz}$. The radar operates in a monostatic configuration with four, five-element Yagi antennas directed along the $0^{\circ}, 90^{\circ} \mathrm{E}, 180^{\circ}$ and $90^{\circ} \mathrm{W}$ meridians. The two-way halfpower halfwidth of the antennas are about $20^{\circ}$ in both azimuth and elevation and the antennas are directed at an elevation angle of $28^{\circ}$. With this configuration we observed about 5000 valid meteor echoes per day.

A comparison between the colocated high resolution $\mathrm{OH}$ spectrometer (Hernandez et al., 1996) and our meteor radar was conducted during 12 consecutive days in the winter of 1995 (Hernandez et al., 1996). This comparison indicated a correlation coefficient between the two sets of measurements of $r=0.82$ at the $99.99 \%$ significance level. An analysis was also conducted for three significant wave periods that were identified during the comparison period. The estimated amplitudes differed by less than $2.5 \mathrm{~ms}^{-1}$ and did not indicate the presence of any systematic bias between the two sets of measurements.

\subsection{Observational issues}

As with any observational instrument, the South Pole meteor radar has it limitations. These limitations typically result either from the scattering mechanism which is being sensed or from system design decisions. The meteor radar which was operational at the South Pole was originally designed to be part of a network of meteor radar systems which were to be deployed across the former Soviet Union and Eastern Europe. As such, the system was designed to be portable and inexpensive to build and operate so that multiple systems could be deployed to study longitudinal structure. To achieve these design criteria a number of choices were made. One of these choices was to utilize compact and lightweight Yagi antennas. These antennas have a wide antenna pattern (about $20^{\circ}$ ). For most applications it would be necessary to use an additional set (two or more) antennas and receivers to locate the position of the observed target and to delineate between multiple targets. However, this additional equipment is unnecessary. The reason is that meteor echoes rarely occur in the same direction at the same time, and that the region where meteors ablate, and hence reflect radio signals, is quite narrow. As a result we can use a wide beam antenna with no height ranging to study the dynamics of the middle atmosphere, at about $95 \mathrm{~km}$. The drawback of this configuration is that no vertical structure information is available and the system is only sensitive to waves with a vertical wavelength in excess of about 20 $\mathrm{km}$. This vertical sensitivity of the radar system and the azimuthal sensitivity are discussed further in the following sections.

2.1.1 Vertical wavelength sensitivity. It is well known that the distribution of radio meteors at very high frequencies (VHF) can be approximated as Gaussian with a halfwidth of about 7 to $10 \mathrm{~km}$ and a peak at $95 \mathrm{~km}$. Because the South Pole meteor radar has no altitude discrimination, the observed hourly averaged winds can be viewed as a Gaussian weighted average of the wave activity around $95 \mathrm{~km}$. This Gaussian weighted average is analogous to filtering the vertically propagating waves using a finite impulse response (FIR) filter with a frequency response which is the Fourier transform of the meteor altitude profile. Therefore to determine the vertical wavelength sensitivity of the meteor radar system we first define the meteor altitude profile

$d(z)=\frac{N}{\sqrt{2 \pi \sigma^{2}}} \mathrm{e}^{-\frac{(z-m)^{2}}{2 \sigma^{2}}}$

where $m$ is the altitude of maximum echoes, $N$ is the total number of echoes, and $\sigma$ is the half-width of the meteor echo distribution at half-maximum. The Fourier transform of $d(z)$ can be expressed as

$D(\lambda)=\frac{N}{\sqrt{2 \pi \sigma^{2}}} \int_{-\infty}^{\infty} \mathrm{e}^{-\frac{(z-m)^{2}}{2 \sigma^{2}}} \mathrm{e}^{-j z \lambda} \mathrm{d} z$

which after completing the square and two changes of variables can be written:

$D(\lambda)=N \mathrm{e}^{-j m \lambda} \mathrm{e}^{-\frac{\sigma^{2} \lambda^{2}}{2}}$,

where $\frac{1}{\lambda}$ is vertical wavelength.

Figure 1 illustrates the vertical wavelength sensitivity for the meteor radar system, where $\mathrm{e}^{-\frac{\sigma^{2} \lambda^{2}}{2}}$ is plotted as a function of vertical wavelength for two values of sigma. These are $\sigma=10$ (solid line) and $\sigma=20$ (dashed line). Clearly evident in Fig. 1 is a reduction in sensitivity for waves with vertical wavelengths less than $40 \mathrm{~km}$. The vertical solid line in Fig. 1 indicates the location of the $3 \mathrm{~dB}$ point, which occurs at a vertical wavelength of $12 \mathrm{~km}$ for $\sigma=10$. The scientific impact of this result is that the amplitude of waves with vertical wavelengths less than $40 \mathrm{~km}$ will be underestimated by the meteor 


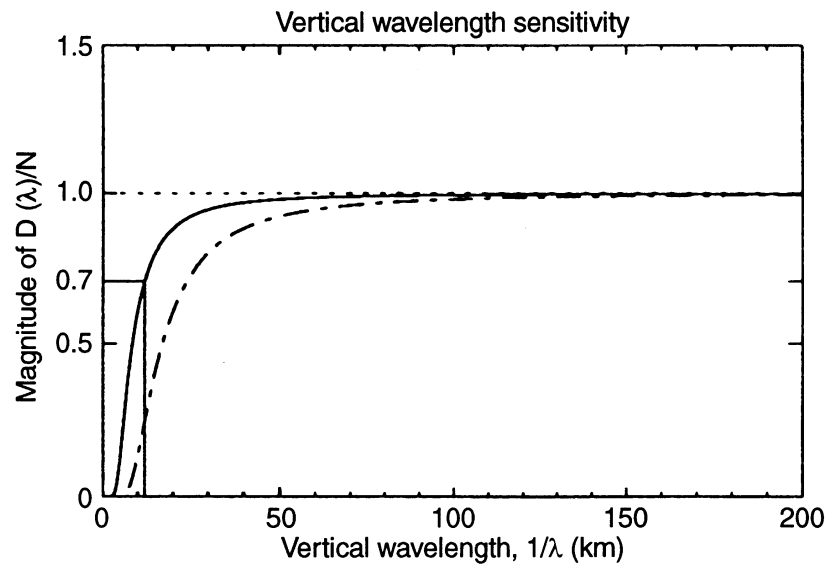

Fig. 1. The sensitivity of the South Pole meteor radar system to waves with differing vertical wavelengths is shown. The solid line (dashed line) indicates the sensitivity assuming a meteor region with a halfwidth of $10 \mathrm{~km}(20 \mathrm{~km})$. The solid vertical line indicates the $3 \mathrm{~dB}$ point for the $10 \mathrm{~km}$ meteor region which occurs at a vertical wavelength of $12 \mathrm{~km}$

radar while waves with vertical wavelengths in excess of $40 \mathrm{~km}$ will experience less than a 5\% underestimation in their amplitudes.

2.1.2 Azimuthal smearing. Typically at midlatitudes an azimuthal beamwidth of $20^{\circ}$ would not be of concern in the extraction of global-scale zonally propagating disturbances. However, at the South Pole where the incremental distance between longitudes becomes small, potential smearing effects due to a nonzero azimuthal beamwidth must be considered. To investigate this issue, consider a time-dependent zonally propagating disturbance which can be expressed as

$v(\lambda, t)=A \cos (\omega t+s \lambda+\phi)$

where, $\omega$ is the radian frequency, $\lambda$ is longitude in radians, $t$ is time, $s$ is zonal wave number and $\phi$ is the phase of the disturbance with an amplitude of $A$. Ideally, one would like to have samples of $v(\lambda, t)$ at $N$ discrete longitudes $\left[\lambda_{0}, \ldots, \lambda_{N-1}\right]$ such that

$v\left(\lambda_{n}, t\right)=A \cos \left(\omega t+s \lambda_{n}+\phi\right)$.

However, this is not possible and in fact we observe on average over some range of longitudes from $\lambda_{n}-\theta$ to $\lambda_{n}+\theta$ where $\theta$ is defined to be the two-way half-power halfwidth of the antenna. Analytically this can be expressed as

$\hat{v}\left(\lambda_{n}, t\right)=\frac{A}{2 \theta} \int_{\lambda_{n}-\theta}^{\lambda_{n}+\theta} \cos (\omega t+s l+\phi) \mathrm{d} l$

and after integration the result is

$\hat{v}\left(\lambda_{n}, t\right)=\frac{2 A}{s \theta} \sin \left(\frac{s \theta}{2}\right) \cos \left(\omega t+s \lambda_{n}+\phi\right)$.
The effect of the finite azimuthal beamwidth can be expressed by taking the ratio of $\hat{v}\left(\lambda_{n}, t\right)$ to $v\left(\lambda_{n}, t\right)$ which is

$\frac{\hat{v}\left(\lambda_{n}, t\right)}{v\left(\lambda_{n}, t\right)}=\frac{2}{s \theta} \sin \left(\frac{s \theta}{2}\right)$.

For the South Pole meteor radar system with a two-way half-power halfwidth of $21^{\circ}$ and zonally propagating disturbance with zonal wave number one $(s=1)$, the net result will be a reduction in the observed amplitude of $0.6 \%$. For a zonal wave number two, the reduction would be $2.2 \%$. However, large-scale zonally propagating disturbances in the meridional wind field are limited to zonal wave number one in the vicinity of the rotational pole (Hernandez et al., 1992, 1995) and this would limit the bias to $0.6 \%$. In cases where waves are present and are propagating around a dynamical rotational pole which is offset from the geographic rotational pole, then waves with zonal wave numbers other than one can exist and in these cases the amplitude bias becomes more significant $(2.2 \%$ for $s=2$ and $5 \%$ for $s=3$ ).

\section{An overview of wave activity over the South Pole}

Using our meteor radar observations it is possible to characterize the wave activity over the South Pole for the whole year. The observed wave spectrum is quite rich and indicates a seasonal asymmetry between the waves which are present during the summer and those present during the winter. To quantify the characteristics of waves present in the meridional wind field over the South Pole we have computed both a discrete spectrogram and an estimate of the wave variance in the 1 to 30 day band of periods for the whole year. These results are discussed in the following sections.

\subsection{Wave spectra}

With observations in four azimuthal directions it is possible to compute frequency wave number spectra. With four equally spaced points in azimuth the Nyquist wave number is 2 . Therefore, using these measurements we can unambiguously determine waves with wave number one both eastward and westward propagating and standing oscillations. It is also possible to estimate the amplitude structure of wave number two disturbances but one cannot distinguish between eastward and westward propagating waves. All higher zonal wave number components will be aliased. However, this aliasing is not expected to be a problem as the first waves to alias onto eastward zonal wave number one are westward zonal wave number 3 and eastward zonal wave number 5 , which are expected to vanish near the rotational pole (Hernandez et al., 1992, 1995).

Figure 2 shows the frequency-wave number spectrogram for the westward propagating wave number one disturbances over the South Pole. This spectrogram results from the computation of consecutive discrete 

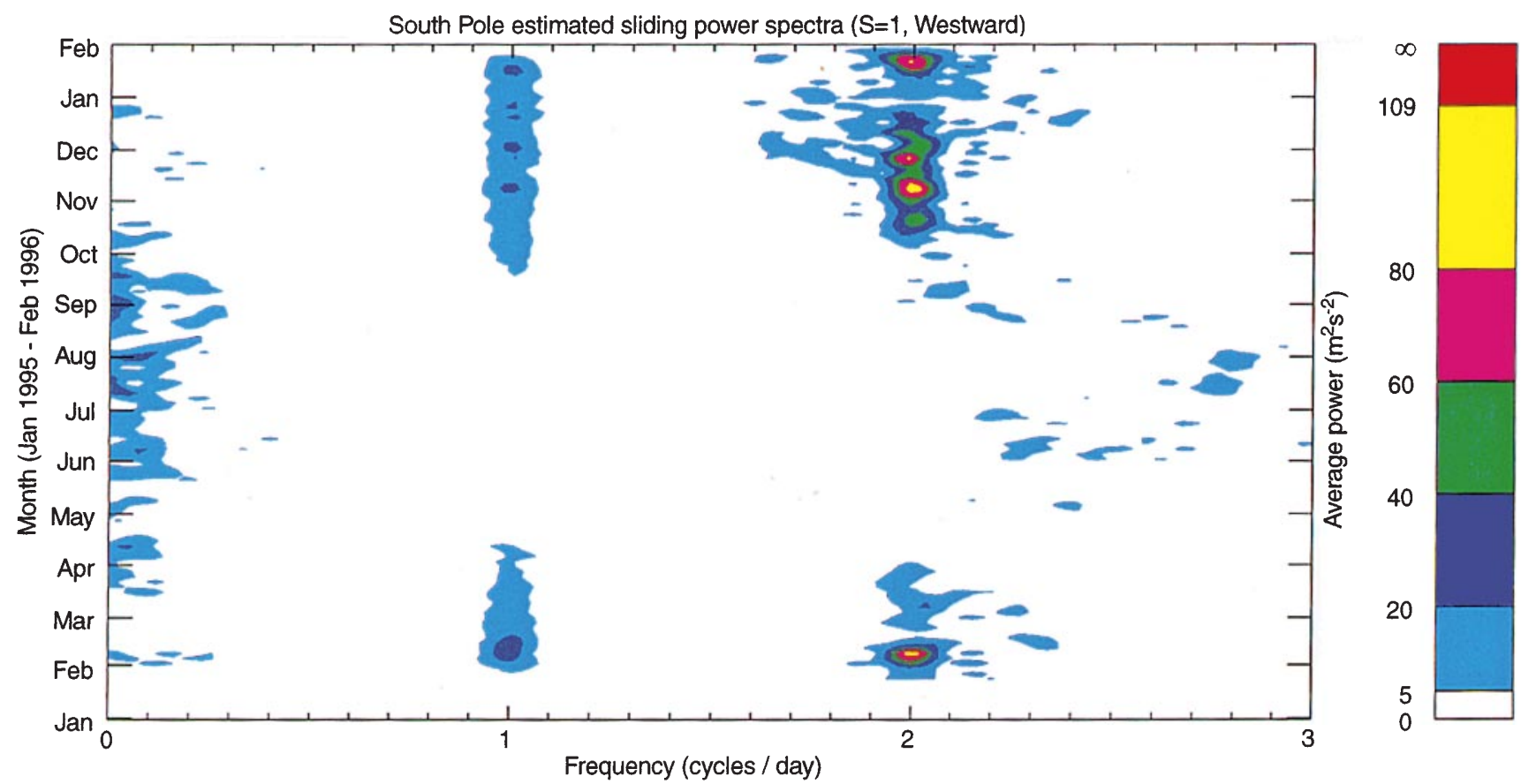

Fig. 2. Shown above is a spectrogram of the westward propagating zonal wave number one disturbances observed over the South Pole in the meridional wind field

periodograms. Each periodogram was computed for ten days of hourly data ( 240 points) and then slid by three days such that there is a seven-day overlap between consecutive periodograms. The periodograms were computed using 1024 points such that the frequency spectra was oversampled by about a factor of five.

Clearly evident in Fig. 2 is a strong $12 \mathrm{~h}$ ( 2 cycles/day) oscillation during the summer months from October through February which wanes during the winter months (Portnyagin et al., 1997b). Also present is a strong $24 \mathrm{~h}$ oscillation with the same type of seasonal behavior as the $12 \mathrm{~h}$ oscillation. During the winter months there is an increase in wave activity for periods longer than 10 days and shorter than $12 \mathrm{~h}$. Previous work by Hernandez et al. (1995) has postulated that a number of these oscillations with periods shorter than $12 \mathrm{~h}$ are related to Lamb waves.

The key used for Figs. 2 and 3 indicates the average power present at a given frequency over the 10 day interval which was analyzed. The scaling for the spectrogram was chosen such that the average power at some frequency $(f)$ is

$$
\begin{aligned}
P(f) & =\frac{1}{T} \int_{-T / 2}^{T / 2}\left|A \mathrm{e}^{-j\left(\frac{2 \pi}{T} t+\phi\right)}\right|^{2} \mathrm{~d} t \\
& =\frac{A^{2}}{2}
\end{aligned}
$$

when integrated over one full wave period. However one should note that if the analysis interval is not divisible by the given wave period then some reduction in the observed average power will occur. This is not related to the choice of scaling but is inherent to Fourier analysis. Additionally, we have not included a level of statistical significance on the plots. The reason for this will be discussed in Sect. 4.2.

Figure 3 shows the frequency-wave number spectrogram for the eastward propagating wave number one disturbances over the South Pole. The temporal and frequency structure of the eastward propagating waves over the South Pole is clearly different from the westward propagating waves as is indicated by comparing Fig. 3 to Fig. 2. The eastward propagating zonal wave number one features exhibit a clear seasonal structure and a lack of wave energy for periods shorter than 2 days. This seasonal structure is described by an increase in the observed frequency of waves which begins in April with periods near 10 days and then migrate towards shorter periods, peaking near a period of 2 days at the winter solstice and then receding to a period near 10 days again around October. Also evident in the spectra are transient increases in the average power near a period of 3 days in June and late August and near a period of 9 days in mid July and early September. These transient wave events will be further discussed in Sect. 4.

Figures 3 and 2 show the time/frequency structure of waves observed over the South Pole for two specific zonal wave numbers. A spectrum which is independent of zonal wave number was presented in Portnyagin et al. (1997b). This spectrogram was computed for each of the four azimuthal directions and then these four spectrograms were averaged to produce a single spectrogram which describes the average wave activity over the South Pole. 


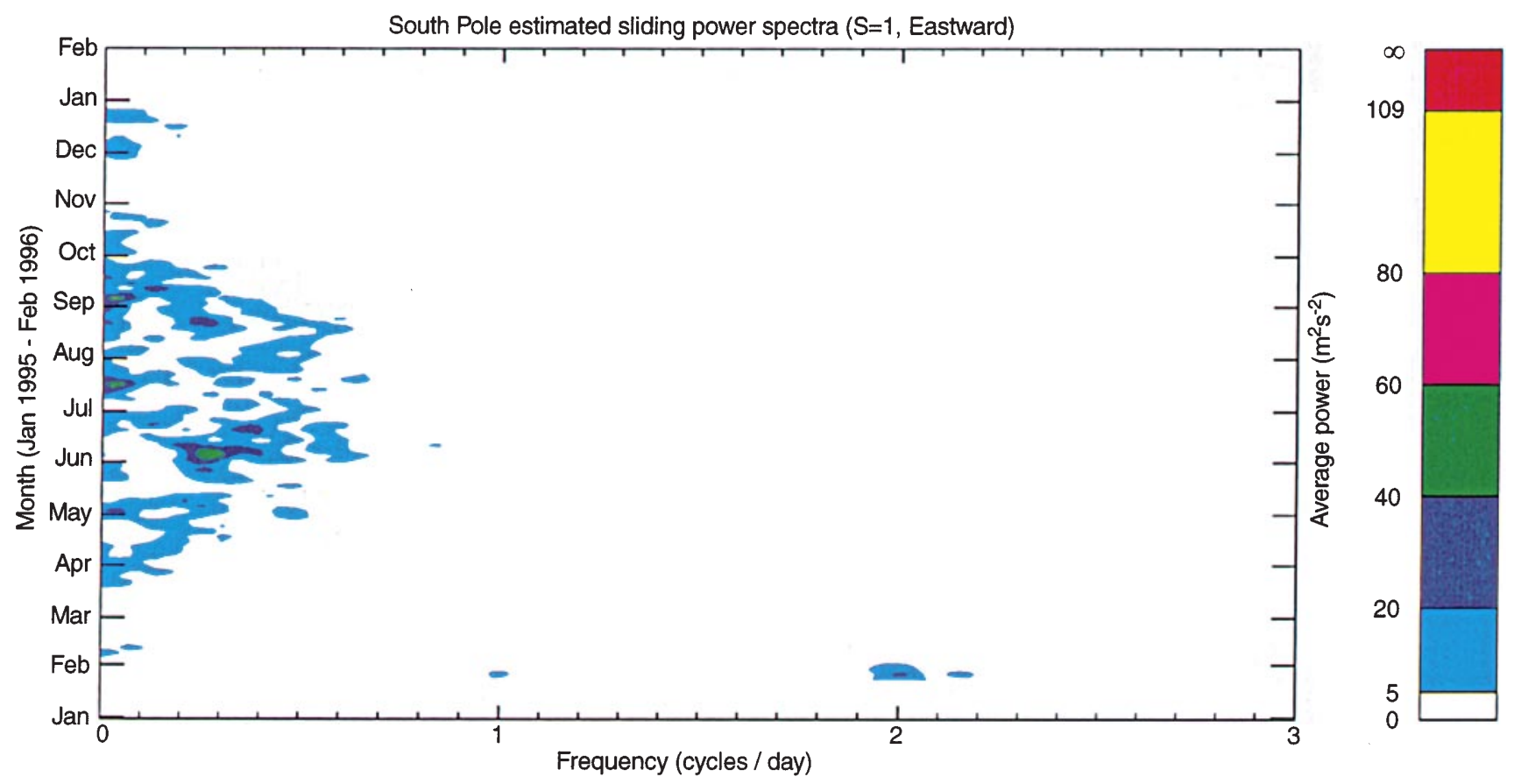

Fig. 3. Shown above is a spectrogram of the eastward propagating zonal wave number one disturbances observed over the South Pole in the meridional wind field

\subsection{Wave variance}

In addition to the frequency-wave number spectrograms, an estimate of the wave variance for all zonal wave numbers and propagation directions present in the meridional wind field was computed. In Fig. 4 is shown the estimated wave variance for periods between 1 and 30 days for the South Pole and Molodezhnaya. The South Pole meridional wind variance was determined by computing the variance of the observed time series over 30 days of data using daily averaged values. In Fig. 4 is an obvious strong annual variation in wave variance for the South Pole with minima in March and October, and a maximum near the winter solstice. The data for April are not shown as there was a strong divergence event observed (Portnyagin et al. 1997a) which included enhanced energy at many frequencies and was not wave-like in character. As this event is not connected with the observed long-period waves it has been removed from Fig. 4. Also shown in Fig. 4 is the zonal and meridional wave variance computed for $6 \mathrm{y}$ of data from Molodezhnaya $\left(68^{\circ} \mathrm{S}\right)$. These variances were again computed for the 1-30 day period range but are only calculated every 10 days. The results for the zonal and meridional wave variances from Molodezhnaya are similar in structure with the zonal values slightly larger than the meridional values throughout the year. There is a large increase in wave variance present during the late summer (JFM) which is primarily due to waves with periods between 2.3 and 3.5 days in both the zonal and meridional directions and is not observed in the South Pole results. Following a decrease in variance values to a near minimum in April the Molodezhnaya variances increase throughout the fall and winter months maxi- mizing in August and September. Then beginning in October the variances begin to decrease. The Molodezhnaya meridional wave variance is less than the South Pole meridional wave variance by as much as $30 \%$ from May through June, but then from July through October the meridional variance observed at the two stations for the 1-30 day periods are comparable and near $80 \mathrm{~m}^{2} \mathrm{~s}^{-2}$. Comparison of the seasonal structure of wave variance for periods between 1 and 30 days appears quite similar

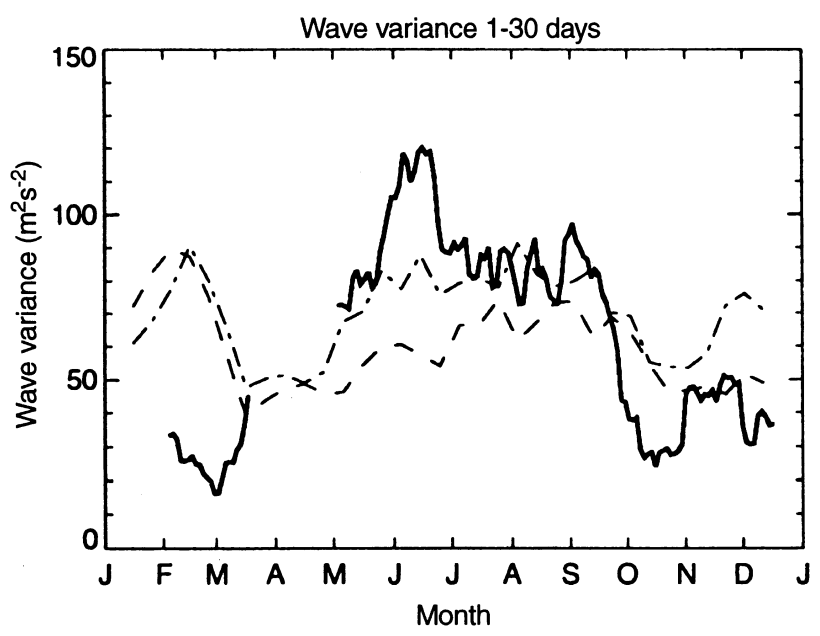

Fig. 4. The total variance $\left(\mathrm{m}^{2} \mathrm{~s}^{-2}\right)$ is shown computed from the South Pole observations during 1995 (solid) for waves with periods between 1 and 30 days. The variance is computed for every other day throughout the year utilizing 30 days of daily averaged data. Also shown are the meridional (dashed) and zonal (dash-dot) estimated wave variances computed using meteor data from Molodezhnaya $\left(68^{\circ} \mathrm{S}\right)$ for 1986 through 1991 
between the South Pole and Molodezhnaya, when one excludes the summer months. These similarities may suggest a degree of latitudinal coherence between the South Pole and Molodezhnaya $\left(68^{\circ} \mathrm{S}\right)$.

\section{Transient wave events}

In the previous section we discussed the seasonal and spectral characteristics of the bulk eastward propagating waves which are present over the South Pole. Clearly present was an increase in eastward propagating planetary waves beginning near the equinox and maximizing around the winter solstice. Embedded in this structure we have observed transient wave events, which are also propagating eastwards. These discrete events possess amplitudes which are 3 to 5 times larger than the underlying background levels of wave activity. In the following sections we will discuss how we identified these waves events, describe their structure and present a theory pertaining to these transient wave events.

\subsection{Constant-Q filterbank}

Rather than using a spectrogram to identify the transient wave events which are present in the data we opted for a constant- $Q$ filterbank. The reason for the choice of a constant- $Q$ filterbank rather than standard Fourier methods is that standard Fourier methods are constrained to possess a frequency resolution which is constant. This frequency resolution is a function of the length of the data segment, or data taper in the case of the spectrogram, which is being utilized for the analysis. For example, if a taper of 10 days in length is used, then 10 cycles of a 1 day oscillation are captured while only one day of a 10 day oscillation is captured. One of the criteria which we used for our identification of transient wave events was that it must be present with significant amplitudes for at least 3 cycles. In the context of our previous example, this means that the wave must be present for 3 days if the period was 1 day and 30 days if the period was 10 days. Clearly, it would be quite difficult to use standard Fourier techniques to determine the presence of transient waves which exist for at least 3 cycles. However, the constant- $Q$ filterbank approach is ideally suited for this type of transient wave analysis because the frequency resolution or filter band width is not constant but rather is a function of the filter center frequency. In fact, the filter $Q$ is defined as $Q=\frac{\omega_{o}}{b w}$, where $\omega_{o}$ is the filter center frequency and $b w$ is the filter band width. Therefore, by definition, if $\mathrm{Q}$ is constant then the band width is linearly related to the center frequency.

Figure 5 shows the frequency response of a constantQ filterbank where the filters are finite impulse response (FIR). It is obvious from Fig. 5 that the filter band widths are not constant and that for higher frequencies the filter band width is larger than for shorter frequencies. Figure 6 shows the impulse response for four filters shown in Fig. 5. These are the filters with center periods of $2.27,4.36,8.36$, and 16.07 days. The $Q$-factor for the

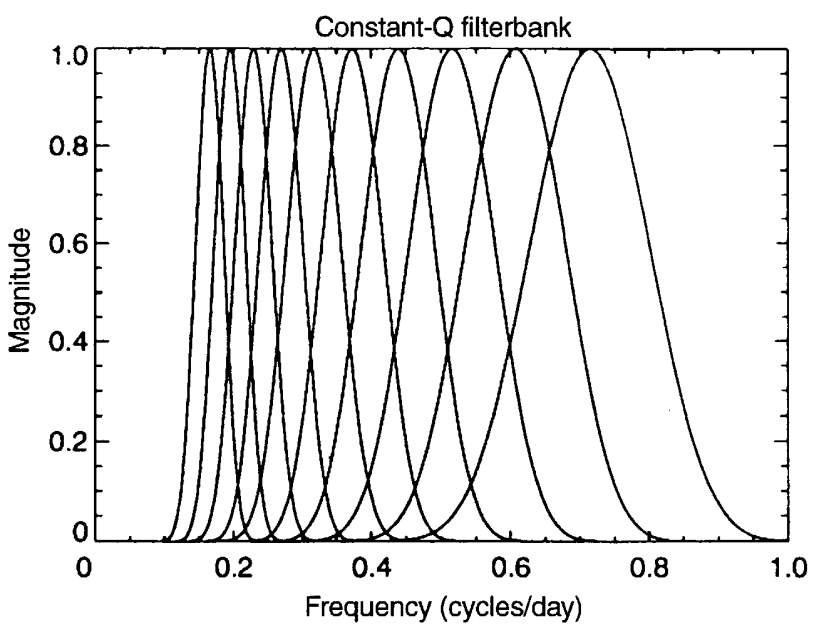

Fig. 5. An example of a constant- $Q$ filterbank

filters was chosen such that approximately 3 cycles at the center period would be present. Notice that the impulse response is a tapered version of a sinusoid at the center period and that the duration of impulse response increases with increasing period. This approach in using a constant- $Q$ filterbank is not significantly different from a Wavelet approach.

\subsection{Estimated significance levels}

While the constant- $Q$ filterbank provides a mechanism which is well suited for the detection of transient waves, we still need a method for determining if an observed transient wave is significant. The standard statistical test for power spectra such as the Chi-Squared and Fischer$\mathrm{F}$ test are applicable, however one must be cautious in the application of these techniques to atmospheric wind
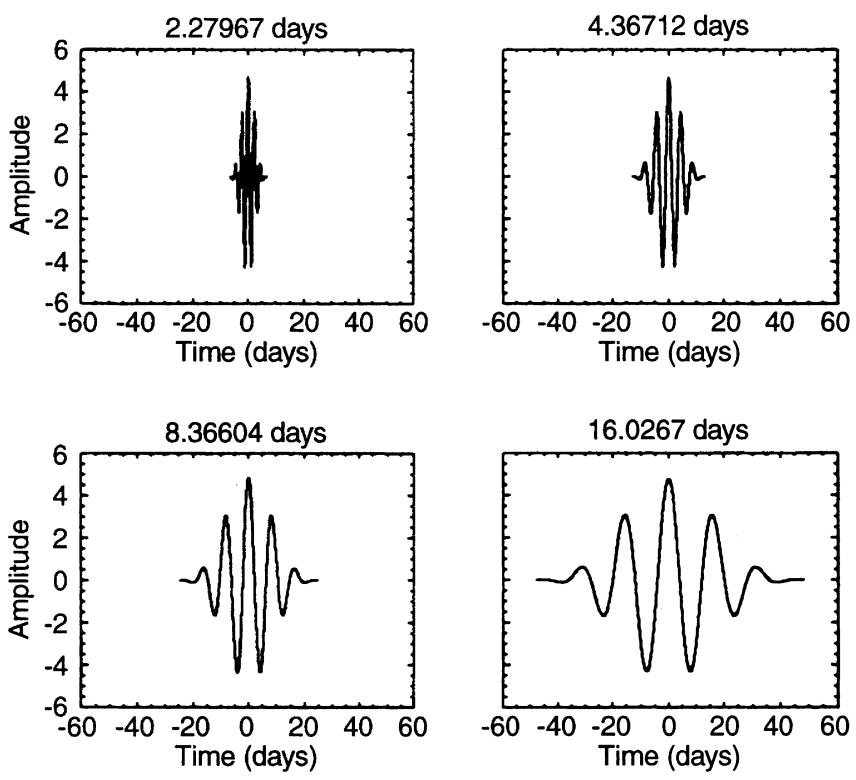

Fig. 6. The time domain filter coefficients for 4 filter bands from the constant- $Q$ filter bank shown in Fig. 5 
and temperature observations. The caveat is that most standard statistical tests, such as those mentioned, assume a model for the observations which is the superposition of narrow band sinusoidal oscillations in background Gaussian noise which is white. The problem is that observed atmospheric temperature and wind fields are NOT white but rather possess some characteristic frequency structure, which is referred to as red. Roughly speaking, a red spectrum is one where the longperiod waves possess more energy than the short-period waves.

Figure 7 is an example of the power spectral density measured from the meteor radar at the South Pole. This spectra was computed by parsing the South Pole data into 11 30-day segments. A simple discrete periodogram was computed for each segment and the 44 segments, 11 for each of the four viewing directions. These were then averaged together to form the composite spectrum shown in Fig. 7. The reason for averaging many individual spectra together is to reduce the variance about the spectral estimate. The $99 \%$ confidence interval for the estimated power spectrum is shown in the upper right hand corner of Fig. 7 and was computed assuming the spectra could be described as a Chi-squared random process with 88 degrees of freedom. The number of degrees of freedom is equal to 2 times the number of independent spectra which were averaged to arrive at the composite spectra. The diagonal line plotted over the spectra in Fig. 7 represents a fit to the spectra between 1 and 30 days assuming a model of the form $A f^{-k}$. This diagonal line represents the estimated mean background structure of the observed power spectrum between periods of 1 and 30 days and will be used in the determination of significant wave events.

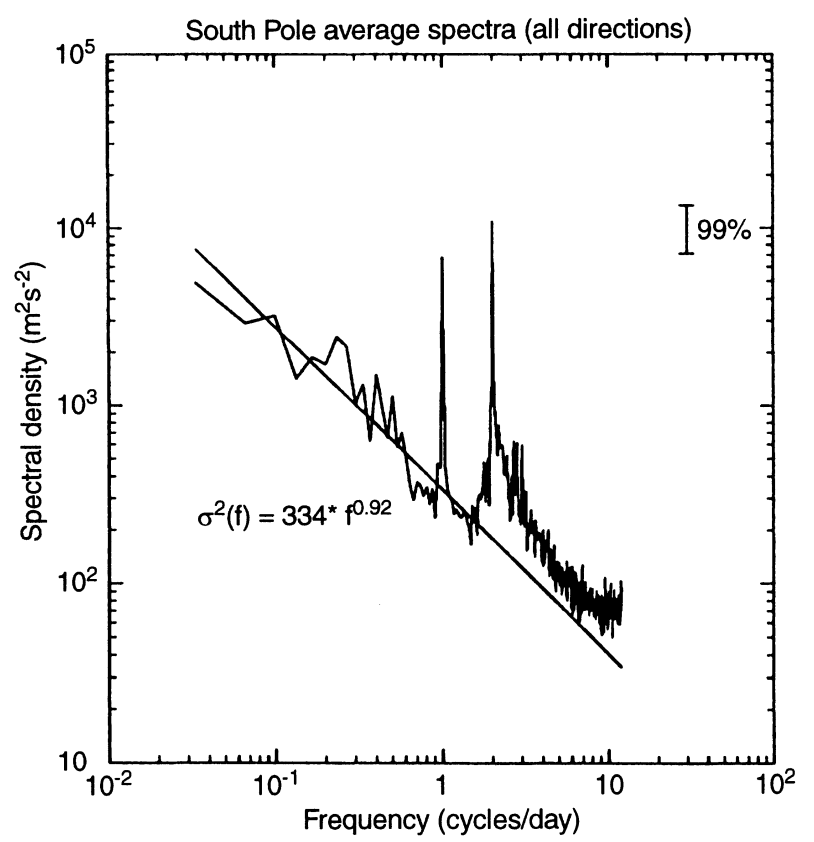

Fig. 7. The estimated South Pole power spectra for periods between $1 \mathrm{~h}$ and 30 days averaged over all four azimuth directions. The solid line is an estimate of the spectral trend between 1 and 30 days assuming a $f^{-k}$ type of dependence
As described, the observed wave spectrum is not white and therefore one cannot assign a single frequency independent significance level to the entire spectrum. Rather, the significance level should reflect the shape of the spectrum in some respect. To this end, a simple model was fit to the spectrum for periods longer than 1 day to arrive at an estimate for the background geophysical noise level, $\sigma^{2}(f)=334 * f^{-0.92}$. Using this level as a benchmark, an observed wave packet will be considered significant if it lies some distance above this line. The distance which an observed wave packet must lies above this line is the significance level.

Because we are using a filterbank rather than a simple periodogram the significance level cannot be derived directly from the spectra, but must include the characteristics of the filters. The Appendix describes the statistical characteristics of a linear time-invariant (LTI) filter. The result is that for a LTI filter the output noise variance $\sigma_{y}^{2}$ is related to the input noise variance $\sigma_{x}^{2}$, assuming a zero mean input time-series, by

$\sigma_{y}^{2}=\sigma_{x}^{2} \sum_{k}|b(k)|^{2}$

where $b(k)$ is the filter impulse response. From this result, $\sigma_{y}^{2}$ provides an estimate of background geophysical noise level where $\sigma_{x}^{2}$ is determined from $\sigma^{2}(f)=334 * f^{-0.92}$, where $f$ is the filter center frequency.

As discussed earlier we are not looking at the actual filter output but rather at the envelope of the filtered wave form. This creates some difficulty in determining the significance level for the observed filtered data because it is not clear how such a transformation will affect the probability density function of the observed filtered data. To address this issue we have performed a Monte-Carlo simulation to determine the cumulative distribution function (CDF) for the observed filtered data. The resulting CDF was obtained by performing 20 simulations each with 10000 independent observations. Figure. 8 shows the CDF which was determined after the Monte-Carlo simulations. In Figure 8 the abscissa indicates the resulting filtered amplitude, while the ordinate is the probability that an amplitude greater than that indicated by the abscissa, using the CDF to map from the ordinate to the abscissa, could be observed. We can now use this CDF to determine significance levels for the filterbank results. The dashed lines in Fig. 8 indicate the location of the $90 \%, 95 \%$, $99 \%$, and $99.9 \%$ significance levels. These values are summarized in Table 1.

Table 1. Significance levels computed via Monte-Carlo simulation and the corresponding filtered amplitudes

\begin{tabular}{ll}
\hline Significance level & Filtered amplitude \\
\hline $90 \%$ & 2.1 \\
$95 \%$ & 2.4 \\
$97.5 \%$ & 2.7 \\
$99 \%$ & 3.0 \\
$99.9 \%$ & 3.6 \\
\hline
\end{tabular}




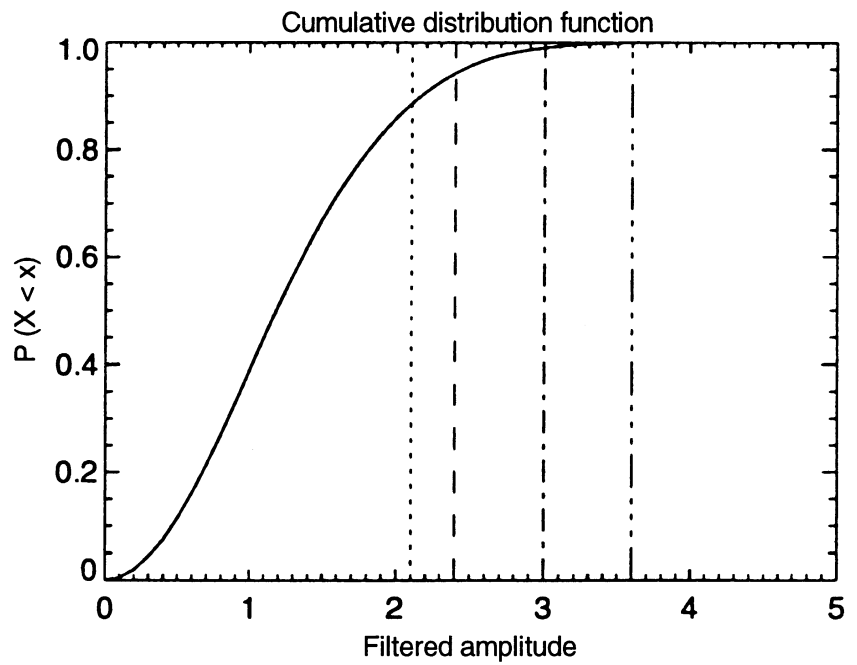

Fig. 8. The cumulative distribution function for the filtered wave amplitudes computed via a Monte-Carlo simulation. The vertical lines indicate the $90 \%$ (dotted), 95\% (dashed), 99\% (dash-dot), and $99.9 \%$ (dash-dot-dot-dot) significance levels

\subsection{Identification of wave events}

To identify long-period wave events which were present in the meteor observations, we utilized the constant- $Q$ filter bank approach, discussed in Sect. 4.1. The $Q$ which was chosen for our filterbank was $0.6 \pi$ and this essentially provides enough band width to account for three cycles of any oscillation at a given wave period. The filterbank consisted of 32 finite impulse response (FIR) filters where the center frequency for the first filter was 1.4 days and the center frequency for consecutive filters was chosen to be the current center frequency minus $25 \%$ of the current filter band width. Such a scheme ensures a reasonably dense coverage of the frequency spectrum.

Figure 9 shows the results of our filterbank analysis for periods between 1.4 and 15 days, from January 1995 to January 1996 , in the $90^{\circ} \mathrm{W}$ viewing direction. Similar plots for the other three viewing directions were constructed but are not displayed here. The actual output of the filters are not shown in Fig. 9 but rather a scaled version of the envelope of the output time series from the filterbank is shown. The envelope of the observed filtered time series can be obtained by demodulating the filtered data using a demodulation frequency equal to the center frequency of the filter or by using a complex valued filterbank and computing the magnitude of the output complex time series. We utilized the second method. The amplitude scale indicates a normalized filtered amplitude which was discussed previously. Amplitudes less than 2, indicating a significance level less than $90 \%$, are not plotted.

Evident in Fig. 9 are a number of wave events which possess normalized filtered amplitudes greater than 4, which would indicate a statistical significance in excess of $99.9 \%$. Clearly these are highly significant peaks. These peaks appear at various times throughout the year

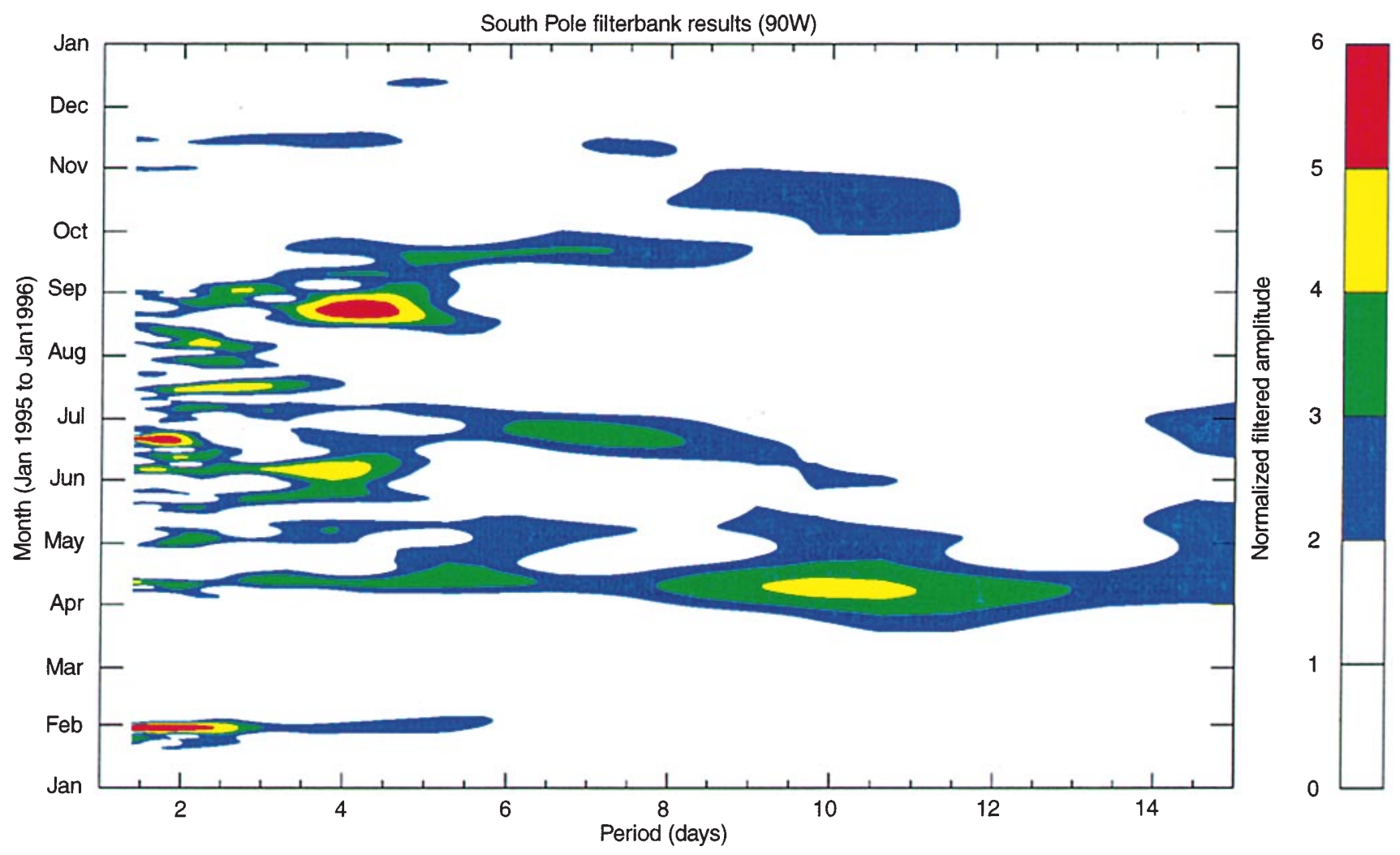

Fig. 9. The result of filtering the South Pole observations from the $90^{\circ}$ west direction using a constant- $Q$ filter bank. The color key is used to indicate statistical significance in terms of $\sigma$ 
and a majority of the peaks exist for periods shorter than 6 days. There is a significant peak present at a period near 10 days; however, this occurred during the large "divergence" event which was observed at the South Pole and should not be considered as a single transient long-period event but rather as part of the wide band "divergence" event which was observed. As a result, we will not consider this peak in further analysis. With the exception of an event observed in February, the remainder of the significant wave events are observed between May and September when there is an increased level of eastward propagating long-period waves observed over the South Pole.

Using the filterbank results from the four observed directions, the eight largest wave events were identified. These wave events were defined to possess a normalized filtered amplitude of at least 4 in one of the four observed directions. The event period was approximated from the center of the largest wave event and the beginning and end of the event was loosely defined as the time when the normalized filtered amplitude fell below 3. Table 2 summarizes the eight wave events which were determined to be "most" significant. All of the wave events chosen began sometime between May and July and possessed normalized filtered amplitudes in excess of 3 for at least 3.8 wave periods.

The four events which most fully describe the characteristics of the wave events observed over the South Pole were selected and plotted in Fig. 10. These were events numbers $1,3,4$, and 5. Each event was filtered using an FIR filter similar to those used in the filterbank described previously, however the filter center
Table 2. Seven significant wave events observed over the South Pole with periods greater than 1 day between January 1995 and January 1996. All events are propagating eastward

\begin{tabular}{lllll}
\hline Number & Period & Start day & End day & Duration \\
\hline 0 & 1.7 & 028 & 034 & 4.1 \\
1 & 3.2 & 152 & 163 & 3.8 \\
2 & 2.5 & 165 & 177 & 5.2 \\
3 & 2.3 & 205 & 213 & 3.9 \\
4 & 2.3 & 216 & 224 & 3.9 \\
5 & 4.1 & 228 & 246 & 4.6 \\
6 & 9.8 & 175 & 235 & 6.2 \\
7 & 5.2 & 240 & 265 & 4.8 \\
\hline
\end{tabular}

frequency was chosen to coincide with the estimated wave period listed in Table 2 for the specified event and the filter band width was chosen such that the filter $Q$ was $0.6 \pi$. This $Q$ is identical to the one used for the filterbank filters. Figure 10 shows the four specified filtered wave events for each one of the four observational directions. The top panel of Fig. 10 shows event 1, the middle panel shows events 3 and 4 which were nearly at the same period, and the bottom panel shows event 5 . The diagonal lines shown in each of the panels of Fig. 10 indicate the phase progression for a zonally propagating eastward wave one disturbance and the shaded regions represent the estimated duration of the event.

Event 1, shown in the top panel of Fig. 10, is observed for about four cycles before the amplitude in the $0^{\circ}$ direction becomes quite small. During the first cycle of this event the wave amplitudes are nearly identical and the phase is consistent with an eastward

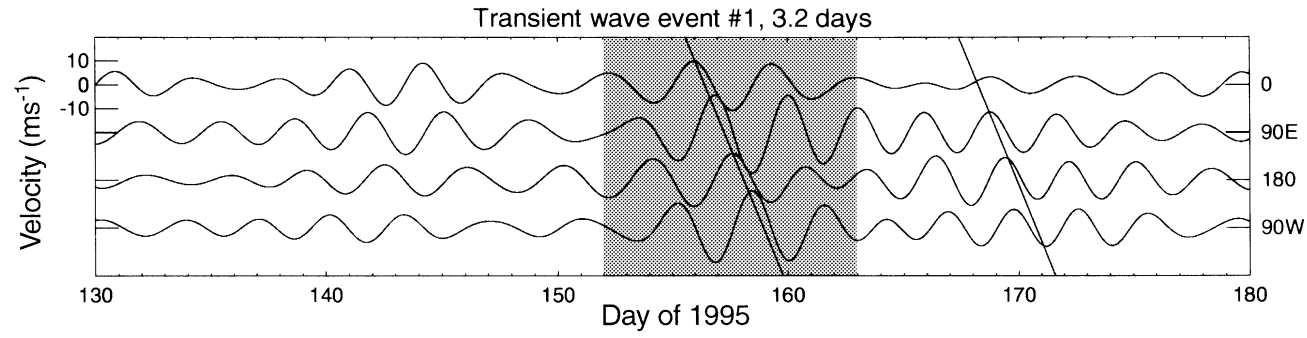

Transient wave event \#3 and \#4, 2.3 days

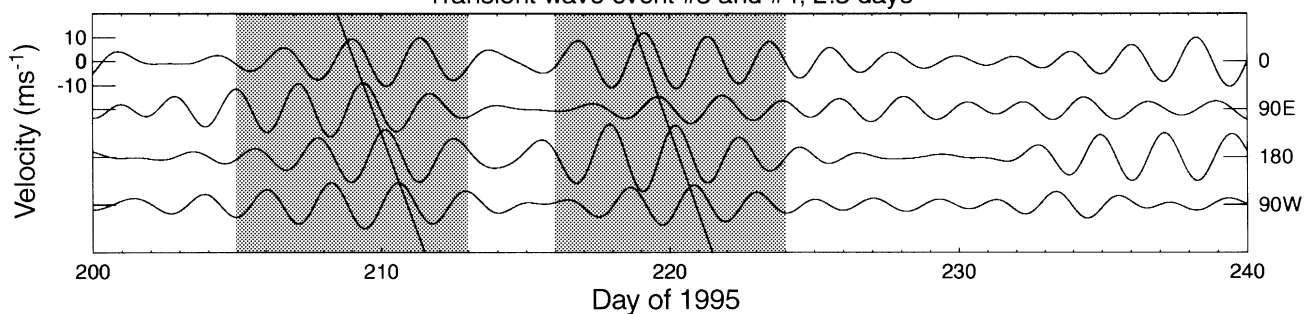

Transient wave event \#5, 4.1 days

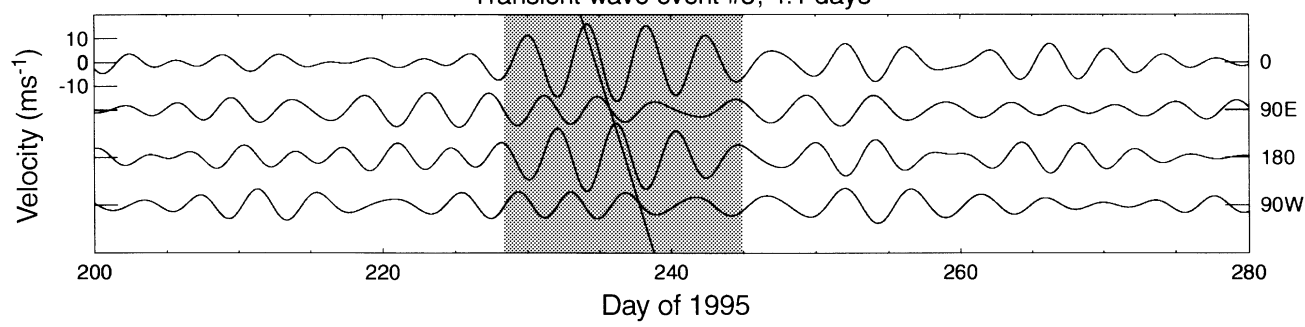

Fig. 10. Filtered time series for wave events $1,3,4$, and 5 . The results for each viewing direction are shown. The shaded region indicates the duration of the wave event 
propagating zonal wave one disturbance. In the ten days which follow this event there are wave amplitudes which attain a significance level in excess of $90 \%$ for three of the four observational directions. Around day 170 the waves observed in the $90^{\circ} \mathrm{E}$ and $180^{\circ}$ directions are still phased such that they are consistent with an eastward propagating wave one disturbance, however the wave observed in the $90^{\circ}$ direction is no longer consistent with this type of behavior.

It is not clear if events 3 and 4 are separate events or if event 4 is a continuation of event 3 . For now, we shall treat these as separate events having the same frequency but separated in time by three days. Event 3 is similar to event 1 in that the amplitudes observed in all four directions possess a similar temporal behavior and the phase relationship between the four directions is consistent with an eastward propagating wave one disturbance. Event 4 is a different matter, where the amplitudes observed in the $0^{\circ}$ and $180^{\circ}$ direction are similar and obviously larger than the amplitudes observed in the $90^{\circ} \mathrm{E}$ and $90^{\circ} \mathrm{W}$ directions. Additionally, the phase relationship is slightly skewed from what would be expected for an eastward propagating wave one disturbance. Event 5 has a duration of 4.6 wave periods or nearly 20 days. This event is similar to event 4 in that decreased wave amplitudes are observed in the $90^{\circ} \mathrm{E}$ and $90^{\circ} \mathrm{W}$ directions. Additionally, the phase structure is shifted from what we would expect for an eastward propagating wave one disturbance.

While Fig. 10 clearly shows the temporal behavior of the four aforementioned wave events, we have also computed least squares fits to each of the wave events. Figure 11 illustrates the results of these fits. The fits were performed to the data for each observational direction separately where the wave period and event duration were taken from Table 2. The phase for these wave events is defined as the time where the wave reaches its maximum amplitude relative to 0UT on the first day fit. In Fig. 11 the phases are plotted on a scale normalized to the assumed wave period. Clearly evident in the phases of all of the fits shown in Fig. 11 is that these waves are eastward propagating, as a stationary wave would have a constant phase structure and a westward propagating wave would have a phase progression orthogonal to the one shown. However, the amplitudes for each of the wave events shown is not constant with respect to the viewing direction. This variability is somewhat confusing as one would expect to observe the same amplitude at each longitude for a zonally propagating disturbance. One can make the case for events 1 , 3 and 4 that there is not a statistically significant difference between the amplitudes as a constant valued amplitude could be chosen which is almost within the errors $(90 \%$ confidence intervals assuming a Gaussian model) for each of the measurements. However, in the case of event 5 one cannot make this argument and clearly there is a difference in the amplitudes measured in the four directions. In fact it appears as though the observations in the $0^{\circ}$ and $180^{\circ}$ direction are similar while the measurements in the orthogonal direction $90^{\circ} \mathrm{E}$ and $90^{\circ} \mathrm{W}$ are similar and nearly a factor of 3
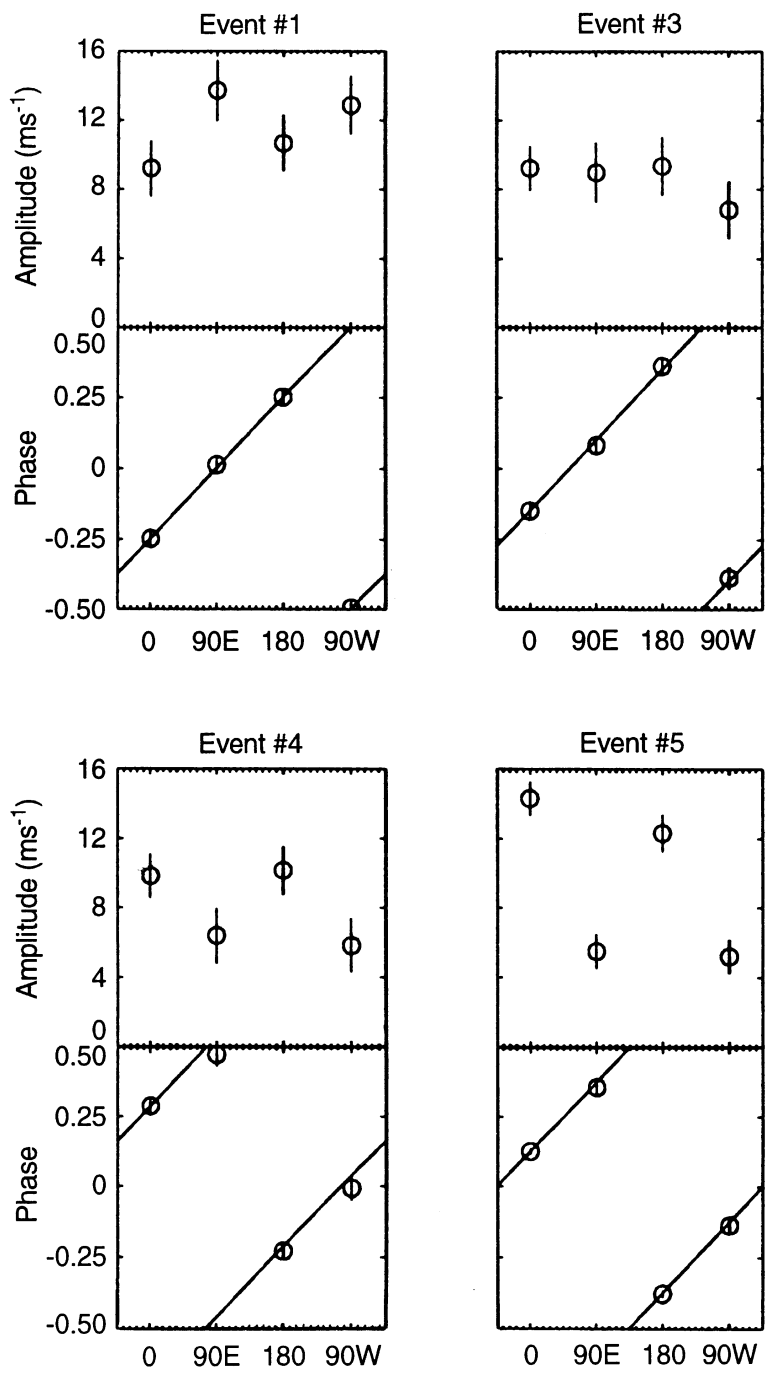

Fig. 11. Results of a linear least squares fit to events $1,3,4$ and 5 . The amplitude (top panel) and phases (bottom panel) are shown for each of the four viewing directions and each event. The error bar for each of the estimated quantities indicates the $90 \%$ confidence interval

smaller than those in the $0 / 180^{\circ}$ direction. One possible explanation for this unusual structure is that the observed wave is not propagating zonally around the geographic pole but rather this wave is propagating around an offset dynamical pole. This hypothesis will be discussed and examined further in the following section.

In addition to the events discussed already we also observed an unusual event in early September 1996, which we have labeled event 7 . A fit to the wave in each of the four directions is shown in Fig. 12. The amplitude of this wave event varies in the four azimuthal directions as was observed for events 4 and 5 but the variation in amplitude and phase from one longitude to the next is quite different than what was observed in the previous wave events. First the phase variation possesses no resemblance to a zonal wave number one phenomena rather the phase is constant in pairs of longitudes where the two sets of longitude pairs are separated in phase by half of a period. The amplitude variation is systematic and can be described by a sinusoidal variation. A similar 


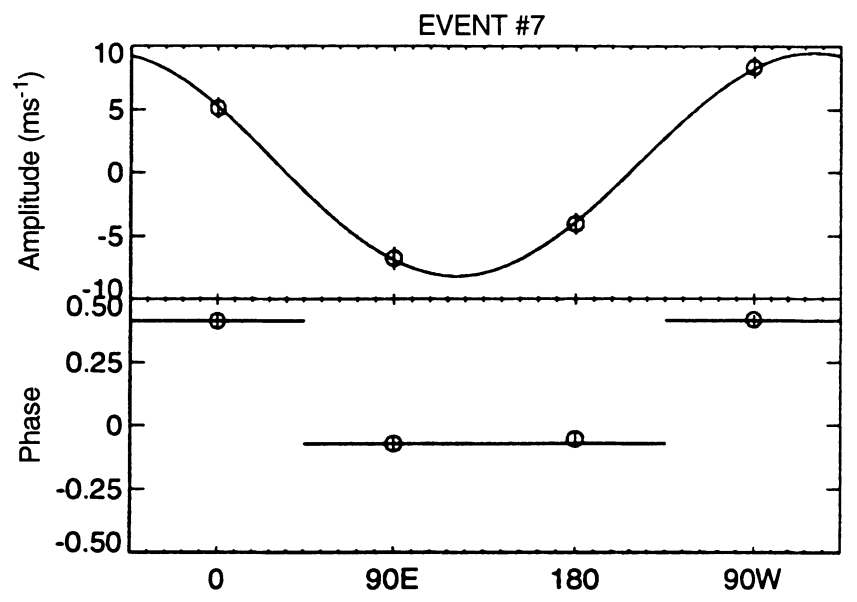

Fig. 12. Results of a linear least squares fit to event 7 . The amplitude (top panel) and phase (bottom panel) are shown for each of the four viewing directions. The error bar for each of the estimated quantities indicates the $90 \%$ confidence interval. Also shown in the top panel is a sinusoidal fit

type of phenomena was observed over the South Pole during August 1996 by Hernandez et al. (1997) which they attributed to a mesospheric standing oscillation. It appears that event 7 is consistent with the structure that Hernandez et al. (1997) observed and may be considered a mesospheric standing oscillation. Event 7 has a period close to 5.2 days $(124 \mathrm{~h})$, an amplitude of $8.9 \pm 0.2 \mathrm{~m} \mathrm{~s}^{-1}$ and is propagating along the $121^{\circ} \mathrm{E}-$ $301^{\circ} \mathrm{E} \pm 1.2^{\circ}$ meridian. During August 12-23, 1996 Hernandez et al. (1997) identified a mesospheric standing oscillation with an amplitude of $13 \mathrm{~m} \mathrm{~s}^{-1}$, a period of $107 \mathrm{~h}$ propagating along the $119^{\circ} \mathrm{E}-299^{\circ} \mathrm{E}$ meridian. These two events would appear to be one and the same with the exception of a shift in time between them. The event observed by Hernandez et al. (1997) occurs 17 days earlier than the event presented herein. We have analyzed the time interval where Hernandez et al. (1997) observed this mesospheric standing oscillation and found the presence of wave activity near a period of $107 \mathrm{~h}$ which maximizes in the $0^{\circ}$ direction with an amplitude of $12.2 \mathrm{~m} \mathrm{~s}^{-1}$. However the wave present during this time, as observed by the meteor radar, has a phase characteristic very similar to event 5 and does not look like event 7 . One possibility for this difference is that the Fabry-Perot spectrometer is observing emissions from a layer centered near $88 \mathrm{~km}$ while the meteor radar is observing meteor reflections from near $95 \mathrm{~km}$.

\section{Discussion}

We have presented evidence from observations over the South Pole taken with a meteor radar that clearly show a strong seasonal variation in the periods and intensity of eastward propagating long period waves. Additionally, we have also localized a number of specific eastward propagating transient long-period wave events during the seasons when a general increase in the eastward wave activity is observed. In the following sections we will discuss the possible mechanisms associated with the observed wave behavior over the South Pole.

\subsection{A mechanism for the observed seasonal behavior}

Based upon the linearized theory for global-scale oscillations in the Earth's atmosphere an oscillation can either be a "free resonant" mode or it must be forced by some mechanism. In an isothermal linearized atmosphere without dissipation and winds such "free resonant" modes can exist in the absence of forcing however in the actual atmosphere some minimal forcing is necessary to excite these modes. Investigation of the dispersion curves for Laplace's tidal equations for an eastward propagating zonal wave number one with wave periods between 2 and 10 days and vertical wavelengths greater than $10 \mathrm{~km}$ only indicate two possible solutions. These solutions are the first symmetric and antisymmetric gravitational modes, which are Kelvin modes typically confined to the equatorial region and to the zonal wind field. Additionally, there do not exist any "free resonant" or Lamb type modes with periods longer than 2 days for eastward propagating zonal wave number one disturbances. Therefore, clearly the observed eastward propagating waves at the South Pole cannot be attributed to free modes and must be related to a forced oscillation. In the case of forced oscillations it is possible to observe waves in the region of forcing or these waves could be forced elsewhere in the atmosphere and propagate into the observing region. Based upon energetic considerations it is typically expected that forced oscillations will be observed in regions of the atmosphere above the level of forcing rather than below.

Previous works (Venne and Stanford, 1982; Lait and Stanford, 1988; Lawrence et al., 1995) have described the presence of waves in the Southern Hemisphere mesosphere and stratosphere with zonal wave numbers one to three and periods of 4, 1.8 and 1.2 days. This 4day wave is an eastward propagating disturbance with a zonal wave number one. Modeling work (Manney et al., 1988; Manney and Randel, 1993) has indicated that this 4-day oscillation is related to an instability, both baroclinic and barotropic, in the winter stratosphere. Fraser et al. (1993) have also found eastward propagating waves present at high southern latitudes using medium frequency radar data from Scott Base $\left(78^{\circ} \mathrm{S}\right)$ and Fabry-Perot spectrometer data from the South Pole. These waves have periods which range from 2 to 4 days and appear to have a zonal wave number of one near the pole.

To investigate the possibility that the observed eastward propagating waves at the South Pole could be related to an instability, we have constructed a mean zonal wind field across the globe for each month of the year and computed the meridional gradient of quasigeostrophic potential vorticity based on this wind field. The meridional gradient of quasi-geostrophic potential vorticity $\left(\bar{q}_{y}\right)$ is a diagnostic tool for the occurrence of 
instabilities, as a change of sign in $\bar{q}_{y}$ is a necessary but not sufficient condition for the onset of unstable wave growth. We have computed $\bar{q}_{v}$ using the equation given by Manney and Randel (1993). Figure 13 shows the mean zonal winds (top) and the resulting $\bar{q}_{y}$ (bottom) for latitudes of $80^{\circ} \mathrm{S}$ and $70^{\circ} \mathrm{S}$. The zonal mean winds were constructed using the semiempirical model of Groves (1985) in the troposphere, geostrophically from MSISE90 temperatures (Hedin, 1991) between 12 and $80 \mathrm{~km}$, and above $80 \mathrm{~km}$ the zonal mean winds were taken from the empirical model of Portnyagin and Solov'yeva (1992a,b). These are the zonal mean wind fields used in global-scale wave model (GSWM) calculation of the diurnal and semidiurnal tides (Hagan et al., 1994, 1995). Clearly evident in the mean zonal wind fields shown in Fig. 13 is a strong eastward circulation which maximizes in the stratosphere in August with an amplitude of $50(80) \mathrm{m} \mathrm{s}^{-1}$ at $80^{\circ} \mathrm{S}\left(70^{\circ} \mathrm{S}\right)$. During the summer months (NDJF) in the mesosphere the circulation reverses to a westward flow. Shown in the bottom panels of Fig. 13 is $\bar{q}_{y}$ for the mean zonal winds discussed above. The shaded regions indicate where $\bar{q}_{y}$ has become negative. At $80^{\circ} \mathrm{S}$ nearly the entire stratosphere and mesosphere possesses a negative $\bar{q}_{y}$ between March and November. As we move to lower latitudes, $70^{\circ} \mathrm{S}$, the structure changes somewhat, where the region of negative $\bar{q}_{y}$ has moved up in the stratosphere and is only present from April to October in the upper stratosphere. One feature which is clearly present in $\bar{q}_{y}$ at $80^{\circ} \mathrm{S}$ and somewhat diminished but still present at $70^{\circ} \mathrm{S}$, is a region of negative $\bar{q}_{y}$ during the winter months near $90 \mathrm{~km}$. One caveat about these results is that they are generated from an empirical climatological model of the zonal mean zonal winds and as such they represent a smoothed average picture of the zonal mean zonal winds during the periods of interested. One can argue that the gradients used to compute $\bar{q}_{y}$ from these data will be underestimated due to the smoothed nature of the climatology. More realistic estimates of $\bar{q}_{y}$ were made by Lawrence and Randel (1996) for June 1975 to June 1978 using the Nimbus-6 PMR data. These results are consistent with our results and indicate a region of negative $\bar{q}_{y}$ present poleward of $70^{\circ} \mathrm{S}$ present during the winter months at 48,65 and $81 \mathrm{~km}$ (other altitudes are not shown). An additional climatology which describes the structure of the Southern Hemisphere middle atmosphere is Koshelkov (1984).

The implication of Fig. 13, is that poleward of $70^{\circ} \mathrm{S}$ the structure of the mean flow is such that waves, which are the result of an instability, could exist and grow. Now, if the waves observed at the South Pole are the result of an instability in the mean flow, then because these waves cannot exist in the absence of forcing, we would only expect to observe these waves during periods when $\bar{q}_{y}$ changes sign. Recall Fig. 3 where the seasonal occurrence of the eastward propagating waves indicates an onset in April with a tendency towards shorterperiod waves through the winter season, and subsiding wave activity in October. From Fig. 13 we see a very similar seasonal dependence of the region of negative $\bar{q}_{y}$
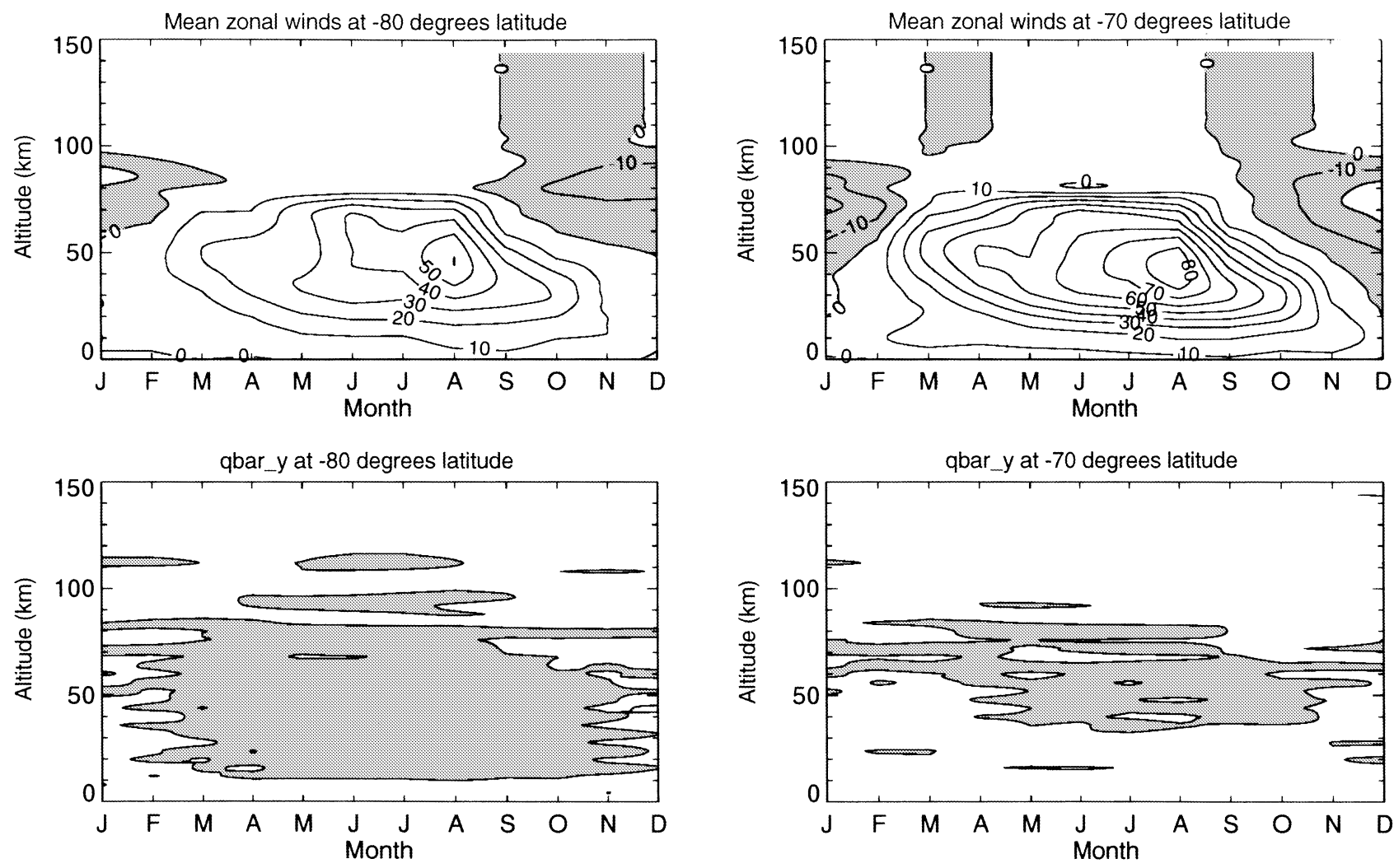

Fig. 13. The mean zonal winds (top) at $80^{\circ} \mathrm{S}$ (left) and $70^{\circ} \mathrm{S}($ right $)$ as a function of month and the latitudinal gradient of quasi-geostrophic potential vorticity (bottom) at $80^{\circ} \mathrm{S}($ left $)$ and $70^{\circ} \mathrm{S}($ right $)$ as a function of month 
during the winter months near $90 \mathrm{~km}$ at $80^{\circ} \mathrm{S}$ and additionally in the stratosphere at $70^{\circ} \mathrm{S}$.

As additional evidence for the connection between an instability near $90 \mathrm{~km}$ and the observed occurrence of eastward propagating waves at the South Pole, Fig. 14 is presented. Figure 14 shows the frequency of the waves which would be excited by the instability near $90 \mathrm{~km}$ at $80^{\circ} \mathrm{S}$. These wave frequencies were estimated using the simple relationship that the phase speed of waves excited by an instability are equal to the mean zonal flow speed in the region where $\bar{q}_{y}$ reverses sign. This relationship was expressed as $m \bar{\omega}-\sigma_{r}=0$ by Manney et al. (1988) and is proven rigorously by Tung (1981) for barotropic instabilities. Assuming a zonal wave number of one, Fig. 14 was constructed using the mean zonal winds at $80^{\circ} \mathrm{S}$ near $90 \mathrm{~km}$ and the aforementioned phased speed relationship. Clearly evident from this figure is a seasonal variation in wave period which is nearly identical to the envelope encompassing the eastward propagating wave spectrogram presented in Fig. 3. Not only is the general seasonal dependence representative of Fig. 3, but the estimated periods are very close in absolute magnitude to what is observed at the South Pole, with a minimum wave period of 2 days observed in July. The minimum wave period of 2 days observed at the South Pole would indicate a mean zonal eastward velocity of $6.4 \mathrm{~m} \mathrm{~s}^{-1}$ for a zonal wave number one disturbance and for increased eastward velocities, shorter wave periods would be expected to occur. From the mean zonal winds shown in Fig. $13\left(80^{\circ} \mathrm{S}\right)$ it can be seen that below $80 \mathrm{~km}$ the zonal mean winds begin to increase rapidly towards the stratopause. This would seem to support a theory that these eastward propagating waves are the result of an instability in the polar mesosphere which is occurring above $80 \mathrm{~km}$. However, as one moves equatorward, the zonal mean wind speed corresponding to a phase speed of $6.4 \mathrm{~m} \mathrm{~s}^{-1}$ increases and it is possible that the observed waves are the result of an instability equatorward and lower in the atmosphere than that postulated earlier. Additional evidence which supports the observation of significant wave amplitudes in the region where an instability is generating waves is the work of Meyer and Forbes (1997). In this paper Meyer and Forbes (1997) excited an unstable 6.5 day wave in

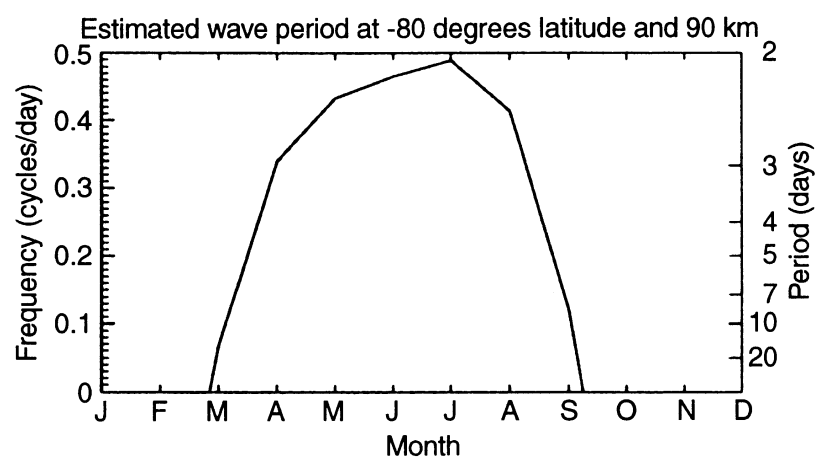

Fig. 14. The instability wave frequency assuming $c=\bar{U}$ for a zonal wave number one disturbance at $80^{\circ} \mathrm{S}$ and $90 \mathrm{~km}$ as a function of month the polar mesosphere and show wave amplitudes in the region of instability which are only $30 \%$ smaller than the maximum amplitudes observed $30 \mathrm{~km}$ higher in the atmosphere. Clearly, without additional observations we cannot rule out either hypothesis.

\subsection{Possible evidence for an offset dynamical pole}

Recall that in Fig. 11 the phases computed for the observed wave events were consistent with what one would expect for an eastward propagating wave with zonal wave number one. However in at least one case, the same cannot be said for the observed amplitude structure. Making measurements with a radar, which can only measure radial velocities, of a wave which is propagating zonally we would expect to observe the same wave feature in each of the four observational directions only delayed by 0.25 of a wave period. Then the resulting fits to this wave feature in each of the four observational directions should give rise to identical amplitudes and phases which are shifted by 0.25 of a wave period relative to the neighboring observations.

Recently, Hernandez et al. (1997) have described a set of optical measurements of mesospheric winds $(\sim 88 \mathrm{~km})$ made near the South Pole during August 1996. During this period Hernandez et al. (1997) observed two distinct wave events in the meridional wind field. These events had periods near $2(51.3 \mathrm{~h})$ and 4 days $(107 \mathrm{~h})$, with peak amplitudes of $10 \mathrm{~ms}^{-1}$. While the amplitude structure is quite similar to the amplitudes which we have observed over the South Pole using the meteor radar, the phase structure of the waves observed by Hernandez et al. (1997) is somewhat different. In particular, for both of these wave event Hernandez et al. (1997) only observed two distinct phases. This coupled with a distinct sinusoidal variation in the observed amplitude with respect to azimuth viewing angle lead Hernandez et al. (1997) to conclude that these observed waves are not zonally propagating but are propagating across the pole. The observed sinusoidal variation in amplitude relative to the azimuthal viewing direction is consistent with a wave propagating over the South Pole rather than zonally around it because the $\mathrm{OH}$ spectrometer, similar to the meteor radar, can only measure radial velocities and hence the observed velocity is the projection of the actual wave velocity projected onto the viewing direction.

Examination of Fig. 11 for events 4 and 5 indicates that the observed amplitudes could not be the result of a simple sinusoidal variation because there are two maxima and two minima observed and the maxima fall between the minima. Clearly, one period of a simple sinusoid would not fit these variations particularly well. Additionally, the observed phase structure is not constant over $180^{\circ}$ of longitude as was observed by Hernandez et al. (1997), and hence we conclude that the wave events presented herein (events 4 and 5) while similar in period, in amplitude and time of occurrence to those presented by Hernandez et al. (1997) are not propagating across the South Pole. Conversely, event 7 
which we have observed is consistent with a mesospheric standing oscillation as presented by Hernandez et al. (1997). If these waves are not propagating zonally around South or across the South Pole then what is the geometry of the observed waves? One possible explanation is that the observed waves are propagating zonally around a dynamical pole which is offset from the geographic pole. If this were the case then the projection of the "true" wave amplitude onto the observational line of sight direction would not result in a simple sinusoidal variation, but would result in a somewhat more complicated geometrical relationship. Figure 15 shows the expected amplitudes and phases for a wave propagating around a dynamical pole which is offset by $400 \mathrm{~km}$ in the $0^{\circ}$ direction. Notice that the amplitudes are large in the $0 / 180^{\circ}$ direction and reduced by at least a factor of 2 in the $90 / 270^{\circ}$ direction. This is quite similar to what was observed for wave events 4 and 5. However, for our simple simulation the expected phases deviate significantly from what is observed. This deviation could be the result of our assumption that the waves are propagating in a circular fashion around this offset dynamical pole. In reality this situation is probably significantly more complicated due reduced phase speeds associated with disturbances at high latitudes which could make these waves more susceptible to interactions with the zonal mean flow.

\section{Conclusions}

The upper mesospheric meteor wind observations over the South Pole presented herein have indicated a significant seasonal variation in wave activity which occurs in this region. Clearly present in the wave spectrum observed over the South Pole is a distinct winter/summer asymmetry with primarily westward propagating waves present during the summer and eastward propagating wave present in the winter. Our analysis has shown that the envelope of eastward propagating waves present during the winter systematically changes with time, where only the longest period waves are observed near the equinoxes and shorter
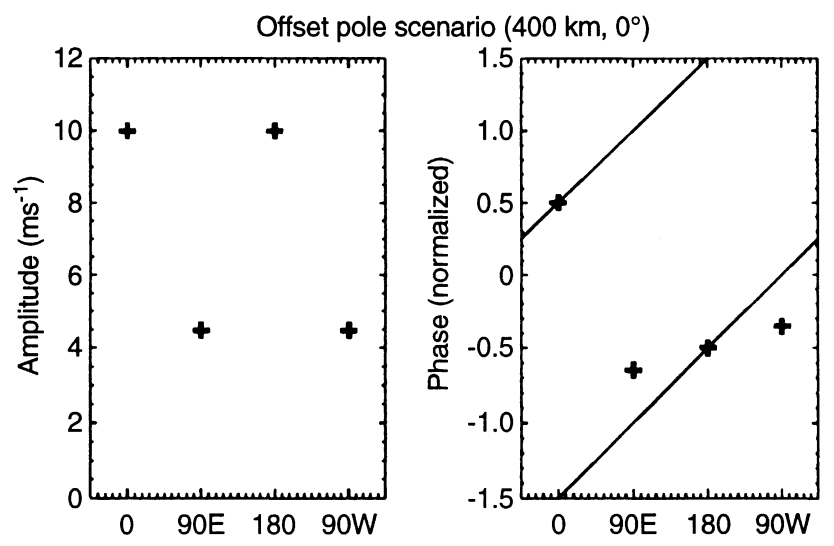

Fig. 15. Amplitude and phase for a $10 \mathrm{~m} \mathrm{~s}^{-1}$ oscillation around a pole offset by $400 \mathrm{~km}$ period waves are present as the winter solstice is approached.

Investigation of the zonal mean wind field at high latitudes indicates that conditions are sufficient for the initiation of unstable wave growth in the upper mesosphere. The seasonal structure of the unstable region and an estimation of the wave periods which may be generated by such an instability are quite similar in structure to the observed seasonal structure of eastward propagating waves observed over the South Pole. This evidence in conjunction with previous modeling efforts and observational studies of the Southern Hemisphere winter stratosphere would indicate that the eastward propagating waves observed over the South Pole could be related to an instability in the upper mesosphere, however a stratospheric source at lower latitudes cannot be ruled out. Additional modeling studies and observations are necessary to further quantify this result.

Embedded in this general increase of eastward propagating wave activity are a number of distinct wave events. Using a constant- $Q$ filterbank and a set of statistical significance criteria the amplitudes, phases and wave periods for these events have been estimated. In a few cases the amplitude structure of the observed waves is inconsistent with the conclusion that the observed wave are propagating zonally around the geographic pole. Rather it appears, in a few cases, that these wave events are propagating around an offset dynamical pole.

Acknowledgements. This work has been supported by the National Science Foundation Office of Polar Programs under grant OPP9320879 and partially supported by the Russian Fund for Fundamental Research under grant N-96-05-64301.

Topical Editor F. Vial thanks B. Lawrence and R.W. Smith for their help in evaluating this paper.

\section{Filtered noise characteristics}

\section{Appendix:}

To begin we ask the question what are the statistical characteristics of a stochastic process which is passed through a linear time-invariant filter. We define $x_{t}$ as a discrete-time stochastic process which is passed through a linear time-invariant (LTI) filter with impulse response $b_{t}$ and the resulting output discrete-time stochastic process is $y_{t}$. From basic linear system theory the output is convolution of the input with the filter impulse response written

$y_{t}=x_{t} * b_{t}$.

The mean value of the output time-series, written $E[y]$, will be $E[x]|B(y)|$, the mean of the input time-series scaled by the filter response at DC. If the filter is not low pass then the resulting output discrete-time stochastic process will be nearly zero mean. The second moment of the output time series, written $E\left[|y|^{2}\right]$, is related to the input time series and the filter frequency response by 
$E\left[|y|^{2}\right]=\frac{1}{2 \pi} \int_{\pi}^{\pi} S_{x x}(\omega)|B(\omega)|^{2} \mathrm{~d} \omega$

where $S_{x x}(\omega)$ is the power spectral density of the input discrete-time stochastic process and $B(\omega)$ is the frequency response of the LTI filter. If the input stochastic process consists of uncorrelated measurements then the power spectral density of this time series is "white" and $S_{x x}(\omega)=\sigma_{x}^{2}$. In this case

$E\left[|y|^{2}\right]=\frac{\sigma_{x}^{2}}{2 \pi} \int_{\pi}^{\pi}|B(\omega)|^{2} \mathrm{~d} \omega$.

Additionally, we can make use of Parseval's relationship which relates the power in the time domain to the power in the frequency domain as

$\sum_{k}|b(k)|^{2}=\frac{1}{2 \pi} \int_{-\pi}^{\pi}|B(\omega)|^{2} \mathrm{~d} \omega$

and as a result

$E\left[|y|^{2}\right]=\sigma_{x}^{2} \sum_{k}|b(k)|^{2}$

where $\mathbf{b}$ is the impulse response of the LTI filter. In the case of a finite impulse response filter (FIR) this is simply the filter coefficients.

\section{References}

Collins, R. L., D. C. Senft, and C. S. Gardner, Observations of a $12 \mathrm{H}$ wave in the mesopause region at the South Pole, Geophys. Res. Lett., 19, 57, 1992.

Forbes, J. M., N. A. Makarov, and Y. I. Portnyagin, First results from the meteor radar at South Pole: a large 12-hour oscillation with zonal wave number one, Geophys. Res. Lett., 22, 3247, 1995.

Fraser, G. J., G. Hernandez, and R. W. Smith, Eastward-moving 2-4 day waves in the winter Antarctic mesosphere, Geophys. Res. Lett., 20, 1547, 1993.

Groves, G. V., A global reference atmosphere from 18 to $80 \mathrm{~km}$, Tech. Rep. AFGL Rep. TR-85-0129, Air Force Geophysical Lab, Bedford, MA, 1985.

Hagan, M. E., J. M. Forbes, and F. Vial, An updated model of migrating tides in the middle atmosphere: initial results and measurement comparisons, in Wind Observations in the Middle Atmosphere, Ed. by M. Geller, CNES, 1994.

Hagan, M. E., J. M. Forbes, and F. Vial, On modeling the migrating solar tides, Geophys. Res. Lett., 22, 893, 1995.
Hedin, A. E., Extensions of the MSIS thermosphere model into the middle and lower atmosphere, J. Geophys. Res., 96, 1159, 1991.

Hernandez, G., R. W. Smith, G. J. Fraser, and W. L. Jones, Largescale waves in the upper-mesosphere at Antarctic high-latitudes, Geophys. Res. Lett., 19, 1347, 1992.

Hernandez, G., G. J. Frazer, and R. W. Smith, Mesospheric 12hour oscillation near South Pole, Antarctica, Geophys. Res. Lett., 20, 1787, 1993.

Hernandez, G., R. W. Smith, and G. J. Fraser, Antarctic highlatitude mesospheric dynamics, Adv. Space Res., 16, 71, 1995.

Hernandez, G., J. M. Forbes, R. W. Smith, Y. Portnyagin, J. F. Booth, and N. Makarov, Simultaneous mesospheric wind measurements near South Pole by optical and meteor radar methods, Geophys. Res. Lett., 23, 1079, 1996.

Hernandez, G., R. W. Smith, J. M. Kelley, G. J. Fraser, and K. C. Clark, Mesospheric standing waves near South Pole, Geophys. Res. Lett., 24, 1987, 1997.

Koshelkov, Y. P., Climatology of the middle atmosphere of the Southern Hemisphere, J. Atmos. Terr. Phys., 46, 781, 1984.

Lait, L. R., and J. L. Stanford, Fast, long-lived features in the polar stratospere, J. Atmos. Sci., 45, 3800, 1988.

Lawrence, B. N., and W. J. Randel, Variability in the mesosphere observed by the Nimbus 6 pressure modulator radiometer, $J$. Geophys. Res., 101, 23475, 1996.

Lawrence, B. N., G. J. Fraser, R. A. Vincent, and A. Phillips, The 4-day wave in the Antarctic mesosphere, J. Geophys. Res., 100, 18899,1995

Manney, G. L., and W. J. Randel, Instability at the winter stratopause: a mechanism for the 4-day wave, J. Atmos. Sci., 50, 3928, 1993.

Manney, G. L., T. R. Nathan, and J. L. Stanford, Barotropic stability of realistic stratospheric jets, J. Atmos. Sci., 45, 2545, 1988.

Meyer, C. K., and J. M. Forbes, A 6.5-day westward propagating planetary wave: Origin and characteristics, J. Geophys. Res., 102, 26173, 1997.

Portnyagin, Y. I., and T. V. Solov'yeva, An empirical model of the meridional wind in the mesopause/lower thermosphere, 1, a mean monthly emperical model, Ross. Meteorol. Gidrol., 10, 28, 1992a.

Portnyagin, Y. I., and T. V. Solov'yeva, An emperical model of the meridional wind in the mesopause/lower thermosphere, 2, height-latitude features of basic components of meridional wind seasonal variations, Ross. Meteorol. Gidrol., 11, 29, 1992 b.

Portnyagin, Y. I., J. M. Forbes, and N. A. Makarov, Unusual characteristics of the lower thermosphere prevailing winds at South Pole, Geophys. Res. Lett., 24, 81, 1997a.

Portnyagin, Y. I., J. M. Forbes, N. A. Makarov, E. G. Merzlyakov, and S. E. Palo, The summertime 12-hour wind oscillation with zonal wave number $s=1$ in the lower thermosphere over the South Pole, Ann. Geophysicae, 1997b, in press.

Tung, K. K., Barotropic instability of zonal flows, J. Atmos. Sci., 38, 308, 1981.

Venne, D. E., and J. L. Stanford, An observational study of highlatitude stratospheric planetary waves in winter, J. Atmos. Sci., 39, 1026, 1982. 